\title{
AUF1 promotes stemness in human mammary epithelial cells through stabilization of the EMT transcription factors TWIST1 and SNAIL1
}

\author{
Manar M. AlAhmari ${ }^{1}$, Huda H. Al-Khalaf ${ }^{1,2,3}$, Falah H. Al-Mohanna ${ }^{4}$, Hazem Ghebeh ${ }^{5}$ and Abdelilah Aboussekhra (1)
}

\begin{abstract}
The AU-rich element RNA-binding protein 1 (AUF1) is an RNA-binding protein, which can both stabilize and destabilize the transcripts of several cancer-related genes. Since epithelial-to-mesenchymal transition (EMT) and the acquisition of cancer stem cell traits are important for cancer onset and progression, we sought to determine the role of AUF1 in these two important processes. We have shown that AUF1 induces EMT and stemness in breast epithelial cells via stabilization of the SNAIL1 and TWIST1 mRNAs, and their consequent upregulation. Indeed, AUF1 binds the transcripts of these two genes at their $3^{\prime}$ UTR and reduces their turnover. Ectopic expression of AUF1 also promoted stemness in mammary epithelial cells, and thereby increased the proportion of cancer stem cells. Importantly, breast cancer cells that ectopically express AUF1 were more efficient in forming orthotopic tumor xenografts in nude mice than their corresponding controls with limiting cell inocula. On the other hand, AUF1 downregulation with specific siRNA inhibited EMT and reduced the stemness features in breast cancer cells. Moreover, AUF1 knockdown sensitized breast cancer cells to the killing effect of cisplatin. Together, these findings provide clear evidence that AUF1 is an important inducer of the EMT process through stabilization of SNAIL1 and TWIST1 and the consequent promotion of breast cancer stem cells. Thereby, AUF1 targeted molecules could constitute efficient therapeutics for breast cancer patients.
\end{abstract}

\section{Introduction}

Breast cancer $(\mathrm{BC})$ is the most common cancer in females worldwide, with nearly $25 \%$ of all new cancer cases and $15 \%$ of all cancer deaths ${ }^{1}$. Considerable advances have been made in the treatment of $\mathrm{BC}$, including local interventions (surgery/radiotherapy) and systemic therapies such as chemo/hormonal and targeted treatments ${ }^{2,3}$. However, $\mathrm{BC}$ recurrence, chemotherapy resistance, and metastasis remain major problems. Recent evidences suggest that $\mathrm{BC}$ recurrence and chemotherapy resistance is mainly due to the presence of a small subpopulation of cells known as BC stem cells (BCSCs) ${ }^{4,5}$. These cells possess stem cell-like features, such as self-

\footnotetext{
Correspondence: Abdelilah Aboussekhra (aboussekhra@kfshrc.edu.sa) 'Department of Molecular Oncology, King Faisal Specialist Hospital and Research Centre, MBC\#03, Riyadh 11211, Saudi Arabia

${ }^{2}$ The National Center for Biotechnology, King Abdulaziz City for Science and Technology, Riyadh 11461, Saudi Arabia

Full list of author information is available at the end of the article
}

renewal and differentiation capacities, which can produce different lineages of cancer cells ${ }^{5,6}$. Furthermore, gaining of stemness traits involves the epithelial-to-mesenchymal transition (EMT) process ${ }^{7,8}$. The activation of such process is under the control of several transcription factors, such as ZEB1 and TWIST1 (refs ${ }^{9-11}$ ).

The control of mRNA stability is one of the most important post-transcriptional mechanism of gene expression regulation ${ }^{10}$. This process is exerted via the binding of RNA-binding proteins (RBPs) to adenylate-uridylate-rich elements (ARE) within the $3^{\prime}$ untranslated region $\left(3^{\prime} \mathrm{UTR}\right)$ of mRNAs ${ }^{12,13}$. The AUbinding factor (AUF1), also called heterogeneous nuclear ribonucleoprotein D (hnRNPD), is one of the best characterized RBPs, which recognizes ARE sequences within different transcripts and controls their stability ${ }^{14}$. Alternative splicing of the AUF1 transcript produces four isoforms, which code for $\mathrm{p} 37, \mathrm{p} 40, \mathrm{p} 42$, and $\mathrm{p} 45$, and all have RNA-binding ability with different affinities ${ }^{15}$. 
The p37 isoform showed the highest affinity for RNA targets as well as pro-carcinogenic effects in transgenic mice $^{15-18}$. The well-known function of AUF1 is the destabilization of mRNAs by forming complexes with other proteins. However, recent studies reported an mRNA-stabilizing role of AUF1 (refs ${ }^{14,19}$ ). Importantly, other studies have shown a considerable increase in the AUF1 expression in tumor cells ${ }^{20}$. Furthermore, AUF1 stabilized the mRNA of the pro-EMT transcription factor ZEB1 in osteosarcoma and thyroid cancer cells ${ }^{21,22}$. These data prompted us to ask whether AUF1 upregulation could promote EMT as well as stemness in mammary epithelial cells. Indeed, we have shown that AUF1 induces EMT and stemness in breast epithelial cells via stabilization of the SNAIL1 and TWIST1 mRNAs, and their consequent upregulation. Our data propose a new strategy for BC treatment, whereby AUF1 inhibition could suppress EMT/stemness, which should promote chemosensitivity and/or prevent cancer relapse.

\section{Results}

Ectopic expression of AUF1 promotes the EMT process in breast epithelial cells

We started the present study by investigating the potential implication of AUF1 in promoting breast carcinogenesis, through inducing EMT in epithelial cells. To this end, we have first ectopically expressed AUF1 in the non-carcinogenic breast epithelial cells (MCF10A) and the luminal breast cancer cells (MCF7). These cells were infected with lentivirus-based vectors either empty (MCF10A-C) (MCF7-C) or bearing p37 ${ }^{\text {AUF1-ORF }}$ (MCF10A-ORF) (MCF7-ORF). Whole-cell lysates were prepared from these cells and the levels of AUF1 and the main EMT markers were assessed by immunoblotting utilizing specific antibodies, while GAPDH and $\beta$-actin were used as internal controls. Figure 1 a shows that p37 ${ }^{\text {AUF1-ORF }}$ increased the level of the four AUF1 isoforms. This could be mediated indirectly through the positive IL-6/STAT3 feedback loop ${ }^{19}$. Concomitantly, the level of the major mesenchymal markers (N-cadherin, Vimentin, SNAIL1, and TWIST1) were also increased, whereas the levels of the epithelial markers EpCAM and E-cadherin were reduced in both cell lines (Fig. 1a). These results were confirmed at the mRNA level by quantitative reverse transcription PCR (qRT-PCR). Indeed, ectopic expression of AUF1 significantly increased the mRNA level of the three EMT-TFs (SNAIL1, ZEB1, and TWIST1) in MCF10A-ORF and MCF7-ORF cells compared to their respective controls (Fig. 1b). In addition to these molecular changes, we have also observed morphological changes in some MCF7 cells expressing p37 ${ }^{\text {AUF1-ORF, }}$ while these phenotypic changes were not obvious for AUF1-expressing MCF10A cells (Fig. 1c). In order to confirm the effect of AUF1 in inducing EMT, we assessed the migration/invasion and proliferation abilities of MCF10A-ORF/MCF10A-C and MCF7-ORF/MCF7-C cells. Thereby, cells were seeded in the upper wells of the CIM plate in the presence of matrigel basement membrane matrix (invasion) or without (migration), while the lower wells contained the appropriate complete medium. Cellular invasion and migration abilities were assessed using the RTCA-DP xCELLigence system for $24 \mathrm{~h}$. Figure $1 \mathrm{~d}$ shows that AUF1 upregulation potently increased the migration and invasion capacities of MCF10A and MCF7 cells as compared to control cells. Next, MCF10A-ORF/ MCF10A-C and MCF7-ORF/MCF7-C cells were added in their appropriate complete medium to the wells of an Eplate and the proliferation rates of such cells were assessed using the RTCA-DP xCELLigence system for $72 \mathrm{~h}$. As expected, MCF10A-ORF and MCF7-ORF cells exhibited higher proliferation rate relative to their respective controls (Fig. 1e). To explore the molecular mechanism underlying the increase in the migration/invasion and proliferation capacities of MCF10A and MCF7 cells expressing $\mathrm{p} 37^{\mathrm{AUF1}-\mathrm{ORF}}$, we investigated the possible involvement of the pro-invasive/migratory and proliferative protein kinase AKT by immunoblotting. Interestingly, the ectopic expression of AUF1 increased the level of the active/phosphorylated form of AKT (Fig. 1a). These findings indicate that AUF1 upregulation promotes EMT in mammary epithelial cells.

AUF1 downregulation inhibits the EMT process in BC cells

To confirm the role of AUF1 in EMT, we have explored the effect of AUF1 downregulation in reversing the EMT process. Thus, AUF1 was knocked-down using specific siRNA and a scrambled sequence was used as control in two triple-negative breast cancer (TNBC) cell lines: MDA-MB-231 (MDA-AUF1si/MDA-C) and BT-20 (BT20-AUF1si/BT20-C) cells. These two cell lines are known to express higher levels of the mesenchymal markers compared to other BC cell lines ${ }^{23}$. AUF1 knockdown in MDA-MB-231 cells upregulated the epithelial markers EpCAM and E-cadherin (4.9-fold) and suppressed the four major mesenchymal markers SNAIL1, TWIST1, ZEB1, and N-cadherin (Fig. 2a). Similar results were obtained for BT-20 cells (Fig. 2a). Figure 2b shows that AUF1 downregulation significantly reduced the mRNA levels of the EMT-TFs SNAIL1, ZEB1, and TWIST1 in MDA-MB-231 and BT-20 cells as compared to their respective controls. These results indicate that AUF1 has an important role in inducing EMT in BC cells. Additionally, following AUF1 downregulation, the migration, and invasion capacities of MDA-MB-231 and BT-20 cells was significantly reduced, suggesting that AUF1 plays a major role in the migratory/invasiveness capacities of BC cells (Fig. 2c, d). Moreover, MDA-AUF1si and BT20-AUF1-si cells exhibited lower proliferation rate 


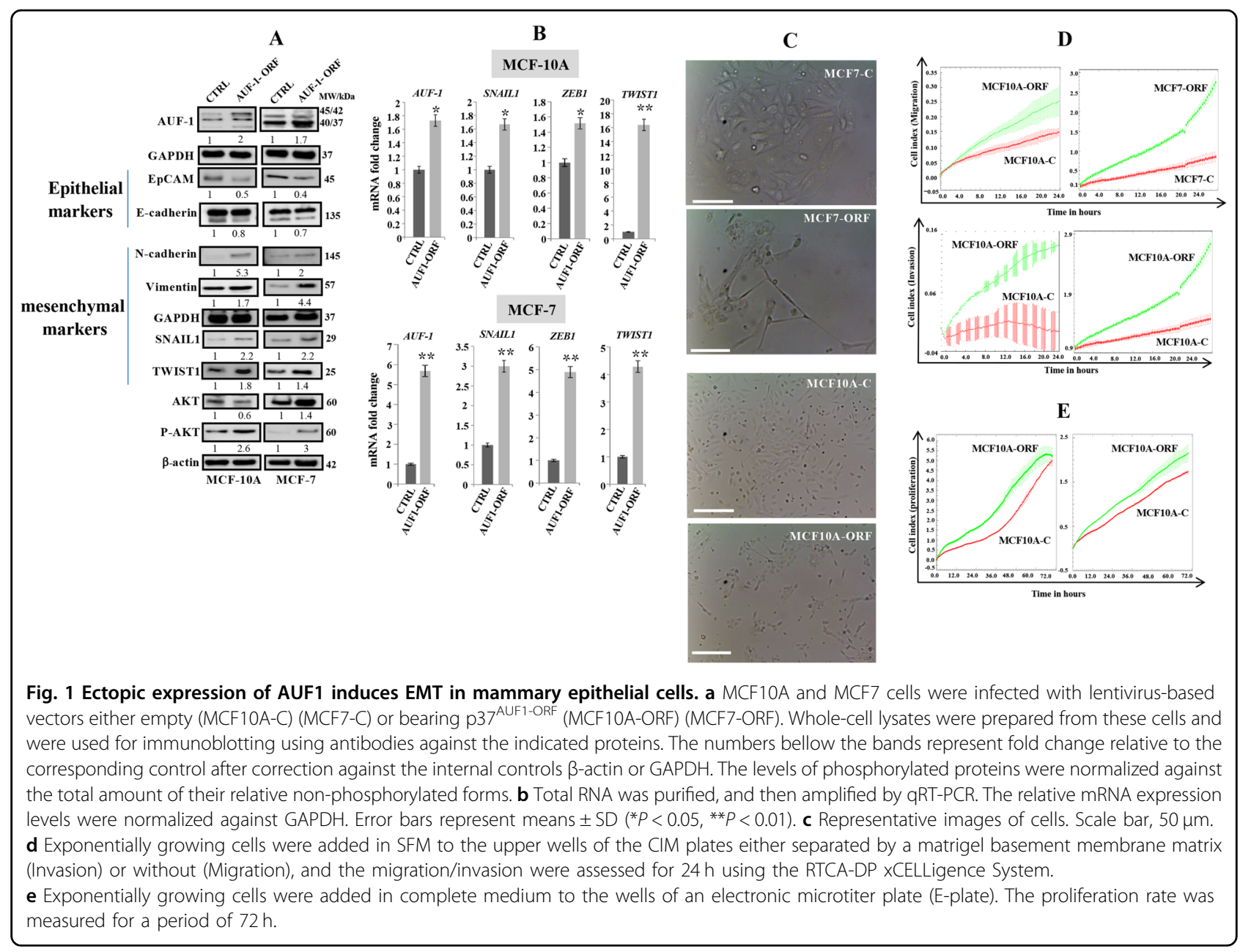

compared to their respective controls (Fig. 2c, d). Similar results were obtained when AUF1 was downregulated with a plasmid bearing specific siRNA pSILENCERAUF1-siRNA that targets all four AUF1 isoforms ${ }^{24}$ (Supplementary Fig. S1). Indeed, AUF1 knockdown promoted the mesenchymal-to-epithelial process and inhibited cellular migration/invasion and proliferation capacities (Supplementary Fig. S1). These results indicate that AUF1 downregulation inhibits the EMT process in $\mathrm{BC}$ cells.

\section{AUF1 binds and stabilizes the TWIST1 and SNAIL1 mRNAs}

To elucidate the molecular mechanism that underlies AUF1-dependent upregulation of SNAIL1 and TWIST1 and the consequent induction of EMT, we sought to investigate the effect of AUF1 on the stability of their transcripts in cells expressing a high level of AUF1 (MCF7-ORF). To this end, MCF7-ORF/MCF7-C cells were treated with the transcription inhibitor actinomycin $\mathrm{D}(5 \mu \mathrm{g} / \mathrm{ml})$, and then were reincubated for different periods of time. Total RNA was purified and amplified with qRT-PCR using specific primers. Figure 3a shows that AUF1 ectopic expression increased the SNAIL1 mRNA half-life. Indeed, while the SNAIL1 mRNA half-life reached $40 \mathrm{~min}$ in MCF7-ORF cells, it was only $15 \mathrm{~min}$ in MCF7-C cells (Fig. 3a). Likewise, AUF1 ectopic expression increased the TWIST1 mRNA half-life from 5 to 8 min (Fig. 3b). On the other hand, AUF1 downregulation by specific siRNA in MDA-MB-231 cells increased the turnover of both TWIST1 and SNAIL1 mRNAs (Fig. 3c, d). These findings indicate that AUF1 stabilizes the SNAIL1 and TWIST1 mRNAs.

\section{AUF1 binds the TWIST1 and SNAIL1 mRNAs at their 3'UTR}

Next, we sought to explore the molecular mechanism underlying AUF1-dependent positive regulation of the mesenchymal markers TWSIT1 and SNAIL1. Since AUF1 is an RBP, we first searched for AUF1- binding site(s) on the $3^{\prime}$ UTR of the TWIST1 and SNAIL1 mRNAs, and we have found two different AUF1-binding sites on the TWIST1 3'UTR, and one on the SNAIL1 3'UTR (Fig. 3e). To prove the binding of AUF1 to the TWIST1 and SNAIL1 3'UTR, biotinylated 3'UTR of each message spanning either the wild type (WT) or the mutated 


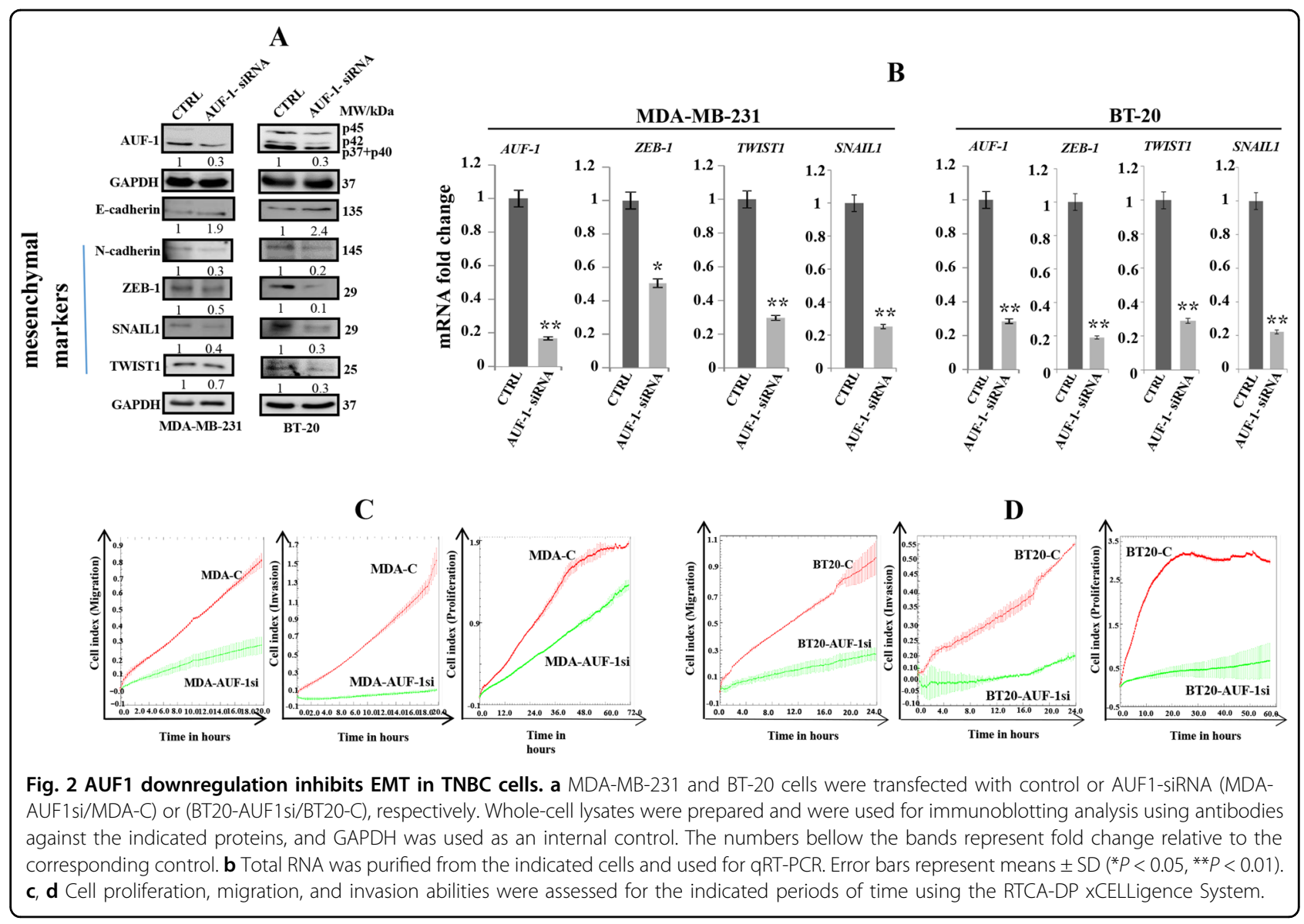

AUF1-binding site were synthesized and incubated with cytoplasmic cellular lysates prepared from MDA-MB-231 cells. The $3^{\prime} \mathrm{UTR} / \mathrm{AUF1}$ ribonucleoprotein complexes were precipitated and the level of AUF1 was assessed by immunoblotting. Figure 3f shows that AUF1 was associated with the two $3^{\prime}$ UTR sequences in control cells. However, when $3^{\prime}$ UTRs bearing mutated AUF1-binding sites were used, AUF1 was almost undetectable (Fig. 3f). This shows the binding of AUF1 to the $3^{\prime} U T R$ sequences of the TWIST1 and SNAIL1 mRNAs in vitro. To confirm this, we investigated the potential contribution of the AUF1-binding sites in the TWIST1 and SNAIL1 mRNA $3^{\prime}$ UTR on the expression of these genes. To this end, wildtype TWIST1 and SNAIL1 3'UTR or the mutated sequences were inserted into a luciferase/Renilla reporter vector and were introduced into MCF7-ORF and MCF7$\mathrm{C}$ cells. The reporter activity fused to the intact sequence of the $3^{\prime}$ UTRs was significantly increased in MCF7-ORF as compared to the control cells (Fig. 3g). Interestingly, the activity was strongly reduced by mutating the putative AUF1-binding site within the $3^{\prime}$ UTR of both mRNAs (Fig. 3g). This further indicates that the effect of AUF1 is mediated through interaction with its seeding sequence in the TWIST1 and SNAIL1 3'UTRs.

\section{Ectopic expression of AUF1 induces stemness in mammary epithelial cells}

The fact that several studies have shown that the induction of EMT leads to the acquisition of stem cell characteristics $^{7,8}$ prompted us to ask whether AUF1mediated EMT induces stemness as well. To address this hypothesis, we tested the effect of AUF1 ectopic expression on the protein level of common stem cell and pluripotency markers in MCF10A-ORF/MCF10A-C and MCF7-ORF/MCF7-C cells. Immunoblotting analysis revealed that the ectopic expression of AUF1 resulted in 2-3-fold increase in the stem cell markers CD44 and ALDH1 (Fig. 4a). On the other hand, the CD24 protein was downregulated 5- and 3.3-fold in MCF10A-ORF and MCF7-ORF cells relative to their respective controls (Fig. 4a). In addition, the protein level of the pluripotency markers KLF4, Oct-4, and Sox2 were also higher in MCF10A-ORF and MCF7-ORF cells compared to their respective controls (Fig. 4a). The AUF1-mediated modulation in the expression of the stemness genes CD24, $C D 44, A L D H 1$, as well as the pluripotency genes OCT-4, $K L F 4, S O X 2$, and $c-M Y C$ was confirmed at the mRNA level using qRT-PCR (Fig. 4b). To confirm the link between AUF1 and the expression of these stemness-related genes, 


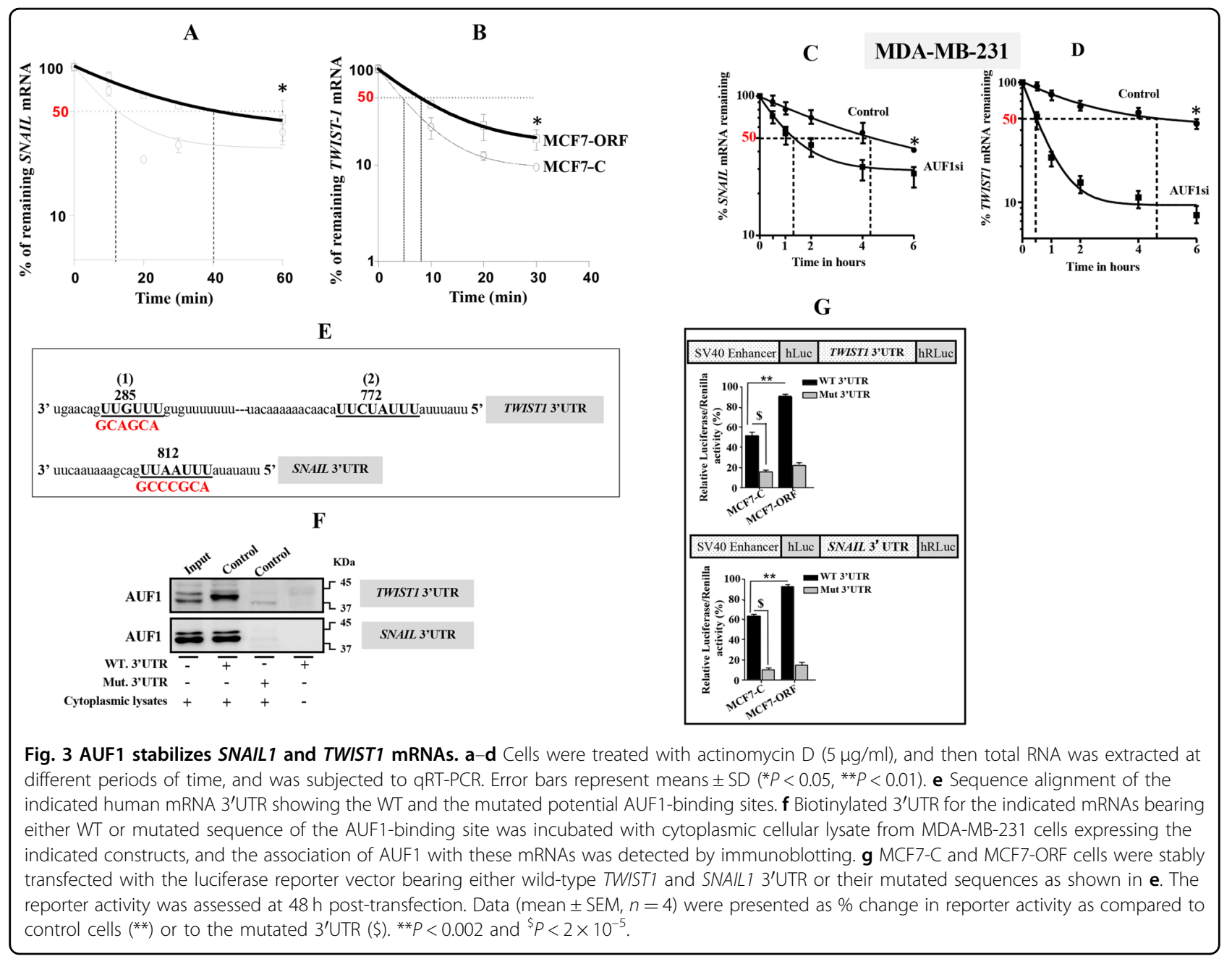

co-staining immunofluorescence experiments were performed. Figure $4 \mathrm{c}$ shows that cells that express a high level of AUF1 stained also positive for CD44 and ALDH1/2. Moreover, the proportion of the two CSC subpopulations: $\mathrm{CD} 44^{\text {high }} / \mathrm{CD} 24^{\text {low }}$ and ALDH ${ }^{\text {high }}$ was quantified by FACS. Figure $4 \mathrm{~d}$ shows that the proportion of $\mathrm{CD} 44^{\text {high }} / \mathrm{CD} 24^{\text {low }}$ subpopulations in MCF10A-ORF and MCF7-ORF cells increased 3- and 3.8-fold compared to their respective controls. Similarly, FACS analysis revealed that MCF10AORF and MCF7-ORF favored the generation of ALDH ${ }^{\text {high }}$ subpopulations in comparison to their respective controls (Fig. 4d).

Next, we investigated the implication of AUF1 in mammosphere formation in MCF7 and MCF10A cells. To this end, MCF10A-ORF/MCF10A-C and MCF7-C/ MCF7-ORF cells were plated at low density into ultra-low attachment plates in the presence of stem cells culture medium. Cells were incubated for 10 days and the formed mammospheres with diameters of $\geq 50 \mu \mathrm{m}$ were counted under the microscope. MCF10A and MCF7 cells ectopically expressing AUF1 demonstrated increase in both size (growth rate) and frequency of spheroids compared to their respective controls (Fig. 4e). Similarly, while control cells did not form colonies (more than $100 \mu \mathrm{m}$ ) in soft agar, MCF7-ORF cells formed big colonies (Fig. 4f). These results indicate that AUF1 promotes $\left(\mathrm{CD} 44^{\text {high }} / \mathrm{CD} 24^{\text {low }} /\right.$ $\mathrm{ALDH}^{\text {high }}$ ) stemness features in breast epithelial cells and induces the formation of BCSCs.

\section{AUF1 ectopic expression induces tumorigenicity in vivo}

To confirm AUF1-dependent formation of BCSC, we injected MCF7-ORF/MCF7-C cells at different concentrations $\left(4 \times 10^{3}, 2 \times 10^{4}, 10^{5}, 5 \times 10^{5} n=3\right.$ for each cell concentration) under the right and left nipples of female nude mice, respectively. After 5 months, while control cells did not form tumors, MCF7-ORF cells generated orthotopic tumor xenografts in all injected animals with volumes proportional to the number of cells injected, reaching a volume of $100-150 \mathrm{~mm}^{3}$ for those injected with $5 \times 10^{5}$ cells (Fig. $4 \mathrm{~g}$ ). These results indicate that AUF1 promotes the formation of CSCs that are responsible for the growth of orthotopic tumor xenografts. 


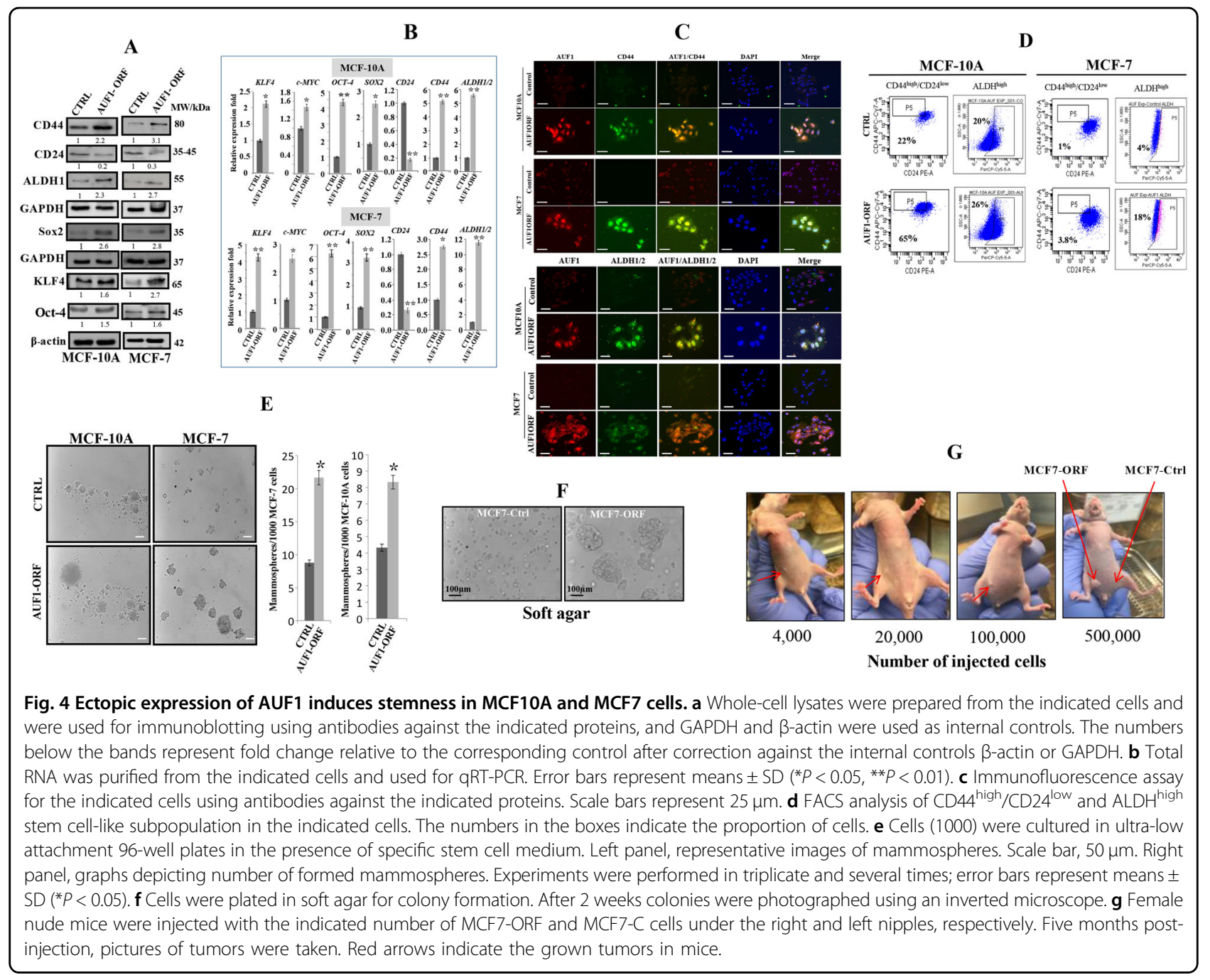

AUF1 promotes stemness in mammary epithelial cells in a TWIST1/SNAIL1-dependent manner

To explore the role of TWIST1 and SNAIL1 in AUF1dependent induction of stemness, we studied the effect of the separate downregulation of these two genes on the expression of the EMT and stem cell markers, in cells ectopically expressing AUF1. Therefore, TWIST1 and SNAIL1 were downregulated using specific shRNA (four different sequences for TWIST1 and three different sequences for SNAIL1) in the MCF7 cells expressing p37 ${ }^{\text {AUF1-ORF }}$ separately. A scrambled sequence was used as control. Figure $5 \mathrm{a}$ shows that all the utilized shRNA sequences knocked-down both TWIST1 and SNAIL1 gene. Subsequently, only two shRNA sequences were utilized to assess the migration/invasion and proliferation abilities of these cells. Figure $5 \mathrm{~b}$ shows that downregulation of TWIST1 or SNAIL1 strongly repressed the migration/invasion as well as the proliferation abilities of these cells, as compared to controls. This indicates that TWIST1 and SNAIL1 are important for AUF1-dependent activation of the migration/invasion and proliferation capacities in MCF7 cells. Interestingly, while downregulation of TWIST1 or SNAIL1 decreased the mRNA levels of the $C D H 2$ and $Z E B 1$ genes, they increased the level of the $C D H 1$ mRNA in cells expressing high level of AUF1 (Fig. 5c). In addition, downregulation of TWIST1 or SNAIL1 decreased the level of the CD44 and ALDH1 mRNAs, while it increased the level of the CD24 mRNA (Fig. 5d). This indicates that the AUF1-dependent modulation in the expression of mammary stem cell-related markers is mediated through TWIST1 and SNAIL1. To confirm this, we investigated the role of these two genes in AUF1-dependent promotion of tumorsphere formation in MCF7 cells. MCF7-Ctl, MCF7-ORF-Ctl as well as MCF7ORF cells expressing either TWIST1 or SNAL1 shRNAs (MCF7-ORF-TWIST1-sh1, MCF7-ORF-SNAIL1-shB) were plated at low density into ultra-low attachment plates in the presence of stem cells culture medium. Cells were incubated for 10 days and the formed mammospheres with diameters of $\geq 50 \mu \mathrm{m}$ were counted under the 


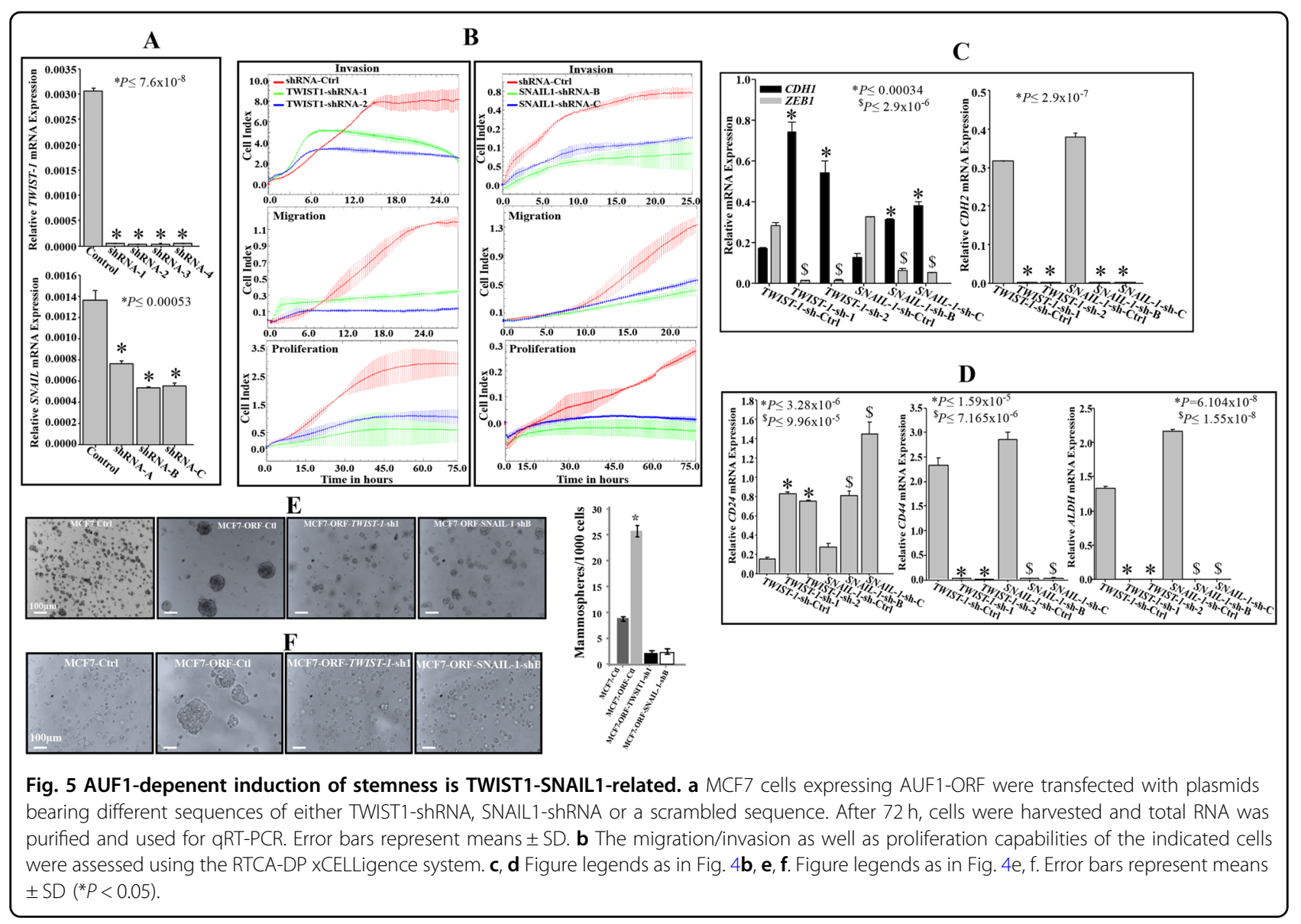

microscope. While control MCF7 cells ectopically expressing $\mathrm{p} 37^{\mathrm{AUF} 1-\mathrm{ORF}}$ demonstrated increase in both size (growth rate) as well as frequency of spheroids as compared to their respective controls, TWIST1 or SNAIL1 downregulation reduced the ability of these cells in forming mammospheres (Fig. 5e). Similarly, while control cells did not form colonies in soft agar, MCF7ORF-Ctl cells formed big colonies; however, no colonies were formed in TWIST1- or SNAIL1-deficient cells (Fig. 5f). This indicates that the AUF1-dependent promotion of stemness in mammalian cells is mediated through TWIST1 and SNAIL1.

\section{AUF1 knockdown decreases stemness in BC cells}

Since AUF1 ectopic expression increased stem cell characteristics, we decided to determine whether the knockdown of AUF1 would reduce stemness. First, we evaluated the protein expression level of the stem cell markers CD44, CD24, and ALDH1 upon AUF1 knockdown in MDA-MB-231 cells. To this end, whole-cell lysates were prepared from MDA-AUF1si/MDA-C cells and were subjected to immunoblotting analysis. The obtained results revealed that AUF1 downregulation significantly reduced the level of CD44 and ALDH1, while increased the expression of CD24, suggesting that AUF1 downregulation inhibits stemness in these cells (Fig. 6a). The AUF1-dependent promotion of stemness was further confirmed by examining the effect of AUF1 downregulation on several pluripotent markers. Figure 6a shows that AUF1 downregulation reduced the protein level of the pluripotency markers KLF4, Oct-4, BMI1, and Sox2. This effect was confirmed at the mRNA level by showing that the downregulation of AUF1 in MDA-MB231 cells significantly reduced the level of KLF4, SOX2, and OCT -4 transcripts. These results indicate that AUF1 knockdown reduces stemness features in $\mathrm{BC}$ cells.

\section{AUF1 downregulation increases cisplatin sensitivity of BC cells}

Since CSCs are known to play a key role in chemotherapy resistance and cancer recurrence, we sought to determine whether AUF1 downregulation would increase MDA-MB-231 cell sensitivity to the chemotherapeutic drug cisplatin. To this end, MDA-AUF1si/MDA-C cells were treated with cisplatin $(20$ and $30 \mu \mathrm{M})$ or DMSO (used as control) for $72 \mathrm{~h}$, and then the WST-1 assay was performed to evaluate the cytotoxic effect. The obtained result indicates that MDA-AUF1si treated with 20 and 
A

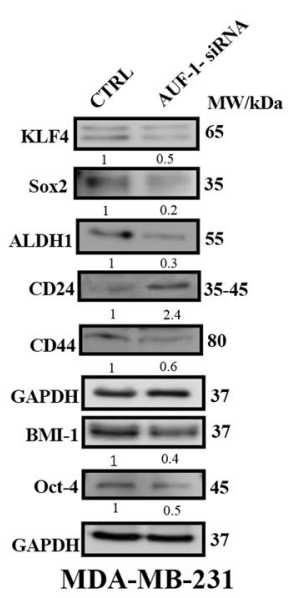

B

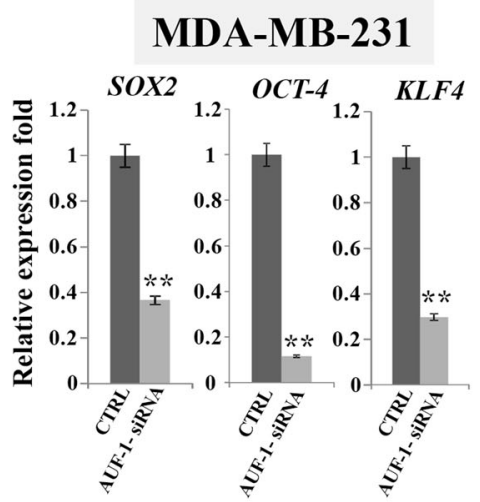

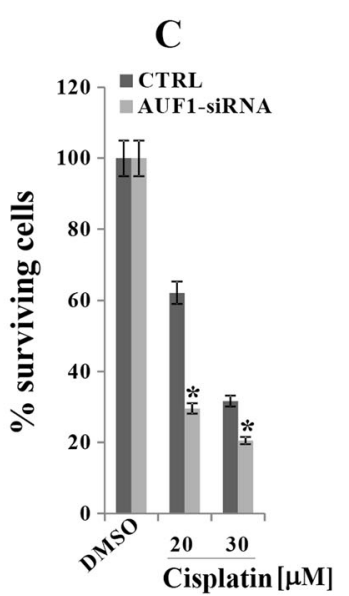

Fig. 6 AUF1 downregulation decreases stemness in TNBC cells and enhances their sensitivity to cisplatin. a MDA-MB-231 cells were transfected with specific AUF1 siRNA (AUF1-siRNA) or a scrambled sequence (CTRL), and then whole-cell lysates were prepared and were used for immunoblotting using antibodies against the indicated proteins. The numbers bellow the bands represent fold change relative to the corresponding control. b Total RNA was purified and was used for qRT-PCR. Error bars represent means $\pm S D(* * P<0.01)$. c Exponentially growing cells were treated either with DMSO (control) or with cisplatin $(20$ and $30 \mu \mathrm{M})$ for $72 \mathrm{~h}$. Cell viability was measured using the WST-1 assay. Error bars represent means \pm SD $\left({ }^{*} P<0.05\right)$.

$30 \mu \mathrm{M}$ of cisplatin resulted in 33 and $11 \%$ increase in cell death compared to controls, respectively (Fig. 6c). These results indicate that AUF1 downregulation sensitizes BC cells to cisplatin.

\section{Discussion}

In the present report we have clearly shown the role of AUF1 in inducing EMT in breast epithelial cells. Indeed, while ectopic expression of AUF1 upregulated the mesenchymal markers, downregulated the epithelial markers, and enhanced the migration/invasion and proliferation capacities of MCF10A and MCF7 cells, AUF1 downregulation in TNBC cells (MDA-MB-231 and BT20) inhibited these EMT features. At the mechanistic level, we have shown that AUF1 upregulated SNAIL1 and TWIST1 through stabilizing their mRNAs. AUF1 binds the AU-rich elements and the potential AUF1-binding sites in the mRNA $3^{\prime}$ UTR of both SNAIL1 and TWIST1. This suggests that AUF1 promotes EMT in breast epithelial cells through post-transcriptional stabilization of these two major EMT-TFs. It has been previously shown that AUF1 stabilizes the EMT inducer ZEB1 in osteosarcoma and thyroid cancer cells ${ }^{21,22}$. Furthermore, other studies have reported the involvement of other RBPs in regulating EMT through stabilizing the $\mathrm{mRNA}$ of the three major EMT-TFs SNAIL1, TWIST1, and ZEB1. For instance, it has been shown that the RBP PTBP3 is an EMT inducer through stabilizing the ZEB1 mRNA and promoting the migration/invasion and proliferation of $\mathrm{BC}$ cells $^{25}$. Another mRNA stability-promoting protein is the human antigen $\mathrm{R}(\mathrm{HuR})$, which is overexpressed in many cancer types. HuR regulates hydrogen peroxide-induced SNAIL1 expression by stabilizing its mRNA, which subsequently enhances cell migration ability through suppressing E-cadherin expression ${ }^{26}$. In contrast, tristetraprolin (TTP) is a tumor suppressor RBP, which is deficient in several cancer types. Indeed, it has been shown that TTP negatively regulates EMT in ovarian cancer cells through binding the SNAIL1 and TWIST1 mRNAs and enhancing their degradation $^{27}$. Together, these findings show that various RBPs are key players in cancer development and progression by post-transcriptionally regulating different EMT-TFs.

Recently, a link between EMT and CSCs has been established, and it became clear that EMT leads to the production of CSCs with stem cell characteristics such as self-renewal ${ }^{7,8}$. Furthermore, the ectopic expression of SNAIL1 or TWIST1 in human mammary epithelial cells induced EMT and also increased the ability of cells to form mammospheres and generate cells with $\mathrm{CD} 44^{\text {high }}$ / $\mathrm{CD} 24^{\text {low }}$ characteristics ${ }^{7}$. In the present study, we have shown that the ectopic expression of p37 AUF1-ORF increases stemness features in MCF10A and MCF7 cells, indicating that AUF1 favors BCSC formation. This has been confirmed in vivo by showing that MCF7-ORF cells have higher ability to form tumors than their corresponding control cells. Indeed, MCF7-ORF xenografts had larger tumors compared to controls. On the other hand, AUF1 downregulation in MDA-MB-231 cells reduced the expression of stem cell markers CD44 and ALDH1, and upregulated CD24 at the protein level. Similarly, the Igf2 mRNA-binding protein 1 (IMP-1), another RBP protein, has been shown to induce loss in 
epithelial characteristics and acquisition of "stem-like" phenotype when overexpressed in colon cancer cells ${ }^{28}$. Indeed, IMP-1 downregulated E-cadherin indorses survival of single tumor cell-derived mammospheres and promoted a significant increase and maintenance of the $\mathrm{CD} 44^{\text {high }} / \mathrm{CD} 24^{\text {low }}$ population ${ }^{28}$. Together, these findings indicate that AUF1 and IMP-1 induce stemness features in non-carcinogenic and carcinogenic cells, and therefore they may play key roles in both the onset and the progression of tumors as well as in their resistance to therapy.

Cisplatin is a well-known platinum-based chemotherapeutic agent, widely used for the treatment of numerous cancers, including BC. Nonetheless, cisplatin has been associated with various side effects as well as drug resistance and tumor recurrence ${ }^{29}$. Growing evidence supports a critical role of CSCs in cancer chemotherapeutic resistance ${ }^{30}$. Intriguingly, several studies have shown that cisplatin induces CSC enrichment in various types of tumors $^{31,32}$. Since AUF1 downregulation reduced the proportion of BCSCs, we hypothesized that this may sensitize BC cells to chemotherapy. Indeed, AUF1 knockdown by specific siRNA in MDA-MB-231 cells increased their sensitivity to cisplatin. This further confirmed the role of AUF1 in inducing stemness in BC, and also indicates the potential importance of targeting AUF1 to enhance the treatment of $\mathrm{BC}$.

Together, these results show that the RNA-binding AUF1 protein plays important roles in breast carcinogenesis through stabilization of the key transcription factors TWIST1 and SNAIL1, and the consequent induction of EMT as well as stemness features. This indicates that, in the era of precision medicine, targeting AUF1 in BC cells could be of great therapeutic value for the hard-to-treat TNBC patients.

\section{Materials and methods}

Cells, cell culture, and reagents

MCF7, MCF10A, MDA-MB-231, and BT-20 cell lines were purchased from ATCC, and were cultured as recommended. Cell lines were authenticated using short tandem repeat profiling by ATCC, propagated, expanded, and frozen immediately into numerous aliquots after arrival. The revived cells were utilized within 10-12 passages and not exceeding a period of 3 months. Cells were regularly screened for mycoplasma contamination using MycoAlert Mycoplasma Detection Kits (Lonza, Basel, Switzerland). All supplements were obtained from Gibco. Cells were maintained at $37^{\circ} \mathrm{C}$ in a humidified incubator with $5 \% \mathrm{CO}_{2}$. Cisplatin was purchased from SigmaAldrich.

\section{Transfection and viral infection}

AUF1-siRNA and control siRNA were obtained from Origene Technologies (Rockville, MD, USA). siRNA sequence: $\quad$ rGrCrCrArUrGrUrCrGrArArGrGrArArCrAr ArUrArUrCrAGC and universal sequence was used as a negative control. In addition, pSILENCER-AUF1-siRNA and control-siRNA plasmids were utilized ${ }^{24}$. The transfections were carried out using the High Perfect reagent (Qiagen), as recommended by the manufacturer. Cells were incubated for 3 days after transfection, recovered, and then were re-cultured for 3 days before collection for subsequent experiments. pLenti-GIII-CMV-hHNRNPDGFP-2A-Puro (Expressing the p37 ${ }^{\text {AUF1 }}$ isoform) (Applied Biological Materials Inc.) and their control plasmids were used at $1 \mu \mathrm{g} / \mathrm{ml}$ each for transfection of 293FT cells. Lentiviral supernatants were collected $48 \mathrm{~h}$ posttransfection. Culture media were removed from the target cells and replaced with the lentiviral supernatant and incubated for $24 \mathrm{~h}$ in the presence of $1 \mu \mathrm{g} / \mathrm{ml}$ polybrene (Sigma-Aldrich). Transduced cells were selected after $48 \mathrm{~h}$ with puromycin. TWSIT1-shRNA and SNAIL1-shRNA and their control plasmids (Origen) were utilized to transfect MCF7-expressing AUF1-ORF using Lipofectamin 3000 following the manufacturer's instructions (Invitrogen).

\section{Cellular lysate preparation and immunoblotting}

This has been performed as previously described ${ }^{33}$. The list of the utilized antibodies is available in the Supplementary Experimental procedures. Each experiment was repeated twice.

\section{Quantification of protein expression level}

Protein signal intensity of each band was determined using ImageQuant TL Software (GE Healthcare). Next, dividing the obtained value of each band by the value of the corresponding internal control allowed a correction of the loading differences. The fold change in the protein levels was determined by dividing the corrected values by that of the control. The levels of the phosphorylated proteins were normalized against the total amount of their relative non-phosphorylated forms.

\section{Determination of mRNA half-life}

Cells were seeded in six-well plates, and were challenged the following day with Actinomycin D $(5 \mu \mathrm{g} / \mathrm{ml})$ for increasing periods of time. Total RNA was then extracted and subjected to qRT-PCR. The one-phase exponential decay curve analysis (GraphPad Prism) was used to assess the mRNA decay kinetics, considering the values at time 0 as $100 \%$. The time corresponding to $50 \%$ remaining mRNA was considered as mRNA half-life. Experiments were performed three times.

\section{Dual-luciferase reporter assay}

Cells were plated at $1 \times 10^{5}$ cells/well on six-well plates and transfected with $3 \mu \mathrm{g}$ of the luciferase/Renilla reporter 
vector containing either human full TWSIT1 or SNAIL1 $3^{\prime}$ UTR, mutated sequence of the AUF1 seed sequence or a control sequence containing no-ARE sequence of the $3^{\prime}$ UTR (GeneCopoeia). Transfection was carried out using Lipofectamin 2000 as recommended by the manufacturer (Invitrogen). At $24 \mathrm{~h}$ post-transfection, cells were seeded in 96-well plate and Firefly and Renilla luciferase activities were consecutively measured using the dual-luciferase assay as recommended by the manufacturer (GeneCopoeia). The Firefly luciferase signal was normalized to the Renilla luciferase signal for each individual analysis. The mean and SEM were calculated from three wells for each $3^{\prime}$ UTR activity and presented as fold change over the nonstimulated control.

\section{Biotin pull-down analysis}

The probes used to prepare biotinylated transcripts spanning the TWIST $3^{\prime} \mathrm{UTR}$ are: (wild type) ACUUGU CAACAAACACAAAAAAA and (mutated) ACUUGU CGCAGCACACAAAAAAA. SNAIL-1 3'UTR are: (wild type) AAGUUAUUUCGUCAAUUAAAUAUAUAA and (mutated) AAGUUAUUUCGUCGCCCGCAUAUAUAA. Biotinylation was performed using the RNA $3^{\prime}$ End Biotinylation kit as instructed by the manufacturer (Thermo Scientific, USA). Cytoplasmic lysates (200 $\mu \mathrm{g}$ per sample) were incubated with $3 \mu \mathrm{g}$ of purified biotinylated transcripts for $30 \mathrm{~min}$ at room temperature, and then the complexes were precipitated with streptavidincoupled Dynabeads (Invitrogen, USA) as previously described $^{34}$. Proteins present in the pull-down material were analyzed by immunoblot analysis. Experiments were repeated twice.

\section{Cell proliferation, migration, and invasion assays}

These assays were performed in a label-free real-time settings using the xCELLigence RTCA Technology (Roche, Germany) that measures impedance changes in a meshwork of interdigitated gold microelectrodes located at the well bottom (E-plate) or at the bottom side of a micro-porous membrane (CIM plate 16) $)^{35,36}$. Cell migration and invasion were assessed as per the manufacturer's instructions. In brief, $2 \times 10^{4}$ cells in serum-free medium were added to the upper wells of the CIM plate coated with a thin layer of Matrigel (BD Biosciences) basement membrane matrix diluted 1:20 in serum-free medium (invasion) or non-coated (migration). Complete medium was used as a chemo-attractant in the lower chambers. Subsequently, the plates were incubated in the RTCA for $24 \mathrm{~h}$ and the impedance value of each well was automatically monitored by the xCELLigence system and expressed as Cell Index (CI) value, which represents cell status based on the measured electrical impedance change divided by a background value. Experiments were performed three times in triplicate.
For the proliferation assay, exponentially growing cells $\left(2 \times 10^{4}\right)$ were seeded in E-plate with complete medium as per the manufacturer's instruction. Cell proliferation was assessed for $48 \mathrm{~h}$. All data were recorded and analyzed by the RTCA software. Cell Index was used to measure the change in the electrical impedance divided by the background value, which represents cell status. Experiments were performed three times in triplicate ${ }^{37}$.

\section{Flow cytometry}

Cells were stained with CD44/CD24 as previously described $^{38}$. Briefly, cells were washed and incubated with CD44 APC-Cy7/CD24 Pacific Blue antibodies (both from Biolegend, USA) for surface staining $\left(30 \mathrm{~min}\right.$ at $\left.4{ }^{\circ} \mathrm{C}\right)$.

\section{Spheroid formation}

Cells were seeded in 96-well ultra-low attachment plate at a density of 1000 viable cells/well. Cells were cultured in 171 medium supplemented with $1 \% \mathrm{ABM}, 2 \% \mathrm{~B}-27$, $20 \mathrm{ng} / \mathrm{ml} \mathrm{EGF,} 500 \mathrm{ng} / \mathrm{ml} \mathrm{HC,} 4 \%$ FBS, and $5 \mu \mathrm{g} / \mathrm{ml}$ insulin. Cells were incubated for 10 days at $37^{\circ} \mathrm{C}$ under $5 \% \mathrm{CO}_{2}$. Mammospheres with a diameter of $\geq 50 \mu \mathrm{m}$ were counted using an OPTIKA light microscope. Experiments were performed three times in triplicate.

\section{Soft agar colony formation assay}

Details are available in Supplementary Experimental Procedures.

\section{Immunofluorescence}

Details are available in Supplementary Experimental Procedures.

\section{Cytotoxicity assay}

Cells $5 \times 10^{3} /$ well were seeded in 96-well plates with appropriate culture media. After cells treatment, WST-1 reagent (Sigma-Aldrich) was added to each well according to the manufacturer's instructions. These experiments were performed in triplicate, and were repeated three times.

\section{Orthotopic tumor xenografts}

Animal experiments were approved by the KFSH\&RC institutional Animal Care and Use Committee (ACUC) and were conducted according to relevant national and international guidelines. Different cell concentrations of MCF7-ORF/MCF7-C cells $\left(4 \times 10^{3}, 2 \times 10^{4}, 10^{5}, 5 \times 10^{5}\right.$ $n=3$ for each cell concentration) were injected under the right and left nipples of 4 weeks female nude mice (NOD. CB17-Prkdcscid/J), respectively. Three animals for each cell concentration was estimated to be adequate for such experiment, and no randomization nor blinding was used. After 5 months, pictures of tumors were taken. 


\section{Statistical analysis}

Statistical analysis was performed by two-sided Student's $t$-test and $P$ values of $\leq 0.05$ were considered as statistically significant.

\section{Acknowledgements}

We are grateful to Dr. Myriam Gorospe for kindly providing us with the pSILENCER-AUF1si plasmid. We are thankful to the Comparative Medicine staff for their help with animal experiments. We also appreciate the continuous support of the Research Center Administration. This work was performed under the RAC proposal \# 2180014, and was supported in totality by King Faisal Specialist Hospital \& Research Center.

\section{Author details}

Department of Molecular Oncology, King Faisal Specialist Hospital and Research Centre, MBC\#03, Riyadh 11211, Saudi Arabia. ${ }^{2}$ The National Center for Biotechnology, King Abdulaziz City for Science and Technology, Riyadh 11461, Saudi Arabia. ${ }^{3}$ KACST-BWH/Harvard Center of Excellence for Biomedicine, Joint Centers of Excellence Program, King Abdulaziz City for Science and Technology (KACST), Riyadh 11461, Saudi Arabia. ${ }^{4}$ Department of Comparative Medicine, King Faisal Specialist Hospital and Research Center, Riyadh 11211, Saudi Arabia. ${ }^{5}$ Stem Cell \& Tissue Re-Engineering Program, King Faisal Specialist Hospital and Research Centre, MBC\#03, Riyadh 11211, Saudi Arabia

\section{Conflict of interest}

The authors declare that they have no conflict of interest.

\section{Ethical approval}

Animal experiments were approved by the KFSH\&RC institutional Animal Care and Use Committee (ACUC) and were conducted according to relevant national and international guidelines outlined in the declaration of Helsenki.

\section{Publisher's note}

Springer Nature remains neutral with regard to jurisdictional claims in published maps and institutional affiliations.

Supplementary Information accompanies this paper at (https://doi.org/ 10.1038/s41389-020-00255-1).

Received: 18 March 2020 Revised: 6 July 2020 Accepted: 16 July 2020 Published online: 05 August 2020

\section{References}

1. Bray, F. et al. Global cancer statistics 2018: GLOBOCAN estimates of incidence and mortality worldwide for 36 cancers in 185 countries. CA Cancer J. Clin. $\mathbf{6 8}$, 394-424 (2018).

2. Ruddy, K. J. \& Ganz, P. A. Treatment of nonmetastatic breast cancer. JAMA https://doi.org/10.1001/jama.2019.3927 (2019).

3. Pazos, M. et al. Recent developments in radiation oncology: an overview of individualised treatment strategies in breast cancer. Breast Care (Basel) 13, 285-291 (2018)

4. Nilendu, P., Kumar, A., Kumar, A., Pal, J. K. \& Sharma, N. K. Breast cancer stem cells as last soldiers eluding therapeutic burn: a hard nut to crack. Int J. Cancer 142, 7-17 (2018).

5. Plaks, V., Kong, N. \& Werb, Z. The cancer stem cell niche: how essential is the niche in regulating stemness of tumor cells? Cell Stem Cell 16, 225-238 (2015).

6. Magee, J. A., Piskounova, E. \& Morrison, S. J. Cancer stem cells: impact, heterogeneity, and uncertainty. Cancer Cell 21, 283-296 (2012).

7. Mani, S. A. et al. The epithelial-mesenchymal transition generates cells with properties of stem cells. Cell 133, 704-715 (2008).

8. Shibue, T. \& Weinberg, R. A. EMT, CSCs, and drug resistance: the mechanistic link and clinical implications. Nat. Rev. Clin. Oncol. https:/doi.org/10.1038/ nrclinonc.2017.44 (2017).

9. Lamouille, S., Xu, J. \& Derynck, R. Molecular mechanisms of epithelialmesenchymal transition. Nat. Rev. Mol. Cell Biol. 15, 178-196 (2014).

10. Puisieux, A., Brabletz, T. \& Caramel, J.Oncogenic roles of EMT-inducing transcription factors. Nat. Cell Biol. 16, 488-494 (2014).
11. Gonzalez, D. M. \& Medici, D. Signaling mechanisms of the epithelialmesenchymal transition. Sci. Signal. 7, re8 (2014).

12. Hentze, M. W., Castello, A., Schwarzl, T. \& Preiss, T. A brave new world of RNAbinding proteins. Nat. Rev. Mol. Cell Biol. 19, 327-341 (2018).

13. Khabar, K. S. Hallmarks of cancer and AU-rich elements. Wiley Interdiscip Rev. RNA 8, https://doi.org/10.1002/wrna.1368 (2017)

14. White, E. J., Matsangos, A. E. \& Wilson, G. M. AUF1 regulation of coding and noncoding RNA. Wiley Interdiscip Rev RNA 8, https://doi.org/10.1002/wrna.1393 (2017).

15. Wagner, B. J., DeMaria, C. T., Sun, Y., Wilson, G. M. \& Brewer, G. Structure and genomic organization of the human AUF1 gene: alternative premRNA splicing generates four protein isoforms. Genomics 48, 195-202 (1998).

16. Zucconi, B. E. et al. Alternatively expressed domains of AU-rich element RNAbinding protein 1 (AUF1) regulate RNA-binding affinity, RNA-induced protein oligomerization, and the local conformation of bound RNA ligands. J. Biol. Chem. 285, 39127-39139 (2010).

17. Moore, A. E., Chenette, D. M., Larkin, L. C. \& Schneider, R. J. Physiological networks and disease functions of RNA-binding protein AUF1. Wiley Interdiscip. Rev. RNA 5, 549-564 (2014).

18. Gouble, A. et al. A new player in oncogenesis: AUF1/hnRNPD overexpression leads to tumorigenesis in transgenic mice. Cancer Res. 62 , 1489-1495 (2002).

19. Hendrayani, S. F., Al-Khalaf, H. H. \& Aboussekhra, A. The cytokine IL-6 reactivates breast stromal fibroblasts through transcription factor STAT3-dependent up-regulation of the RNA-binding protein AUF1. J. Biol. Chem. 289, 30962-30976 (2014)

20. Zucconi, B. E. \& Wilson, G. M. Modulation of neoplastic gene regulatory pathways by the RNA-binding factor AUF1. Front Biosci. (Landmark Ed.) 16, 2307-2325 (2011).

21. Al-Khalaf, H. H. \& Aboussekhra, A. MicroRNA-141 and microRNA-146b-5p inhibit the prometastatic mesenchymal characteristics through the RNAbinding protein AUF1 targeting the transcription factor ZEB1 and the protein kinase AKT. J. Biol. Chem. 289, 31433-31447 (2014).

22. Li, S. et al. Induction of epithelial-mesenchymal transition (EMT) by Beclin 1 knockdown via posttranscriptional upregulation of ZEB1 in thyroid cancer cells. Oncotarget 7, 70364-70377 (2016).

23. Malhotra, G. K., Zhao, X., Band, H. \& Band, V. Histological, molecular and functional subtypes of breast cancers. Cancer Biol. Ther. 10, 955-960 (2010).

24. Wang, W., Martindale, J. L., Yang, X., Chrest, F. J. \& Gorospe, M. Increased stability of the p16 mRNA with replicative senescence. EMBO Rep. 6, 158-164 (2005).

25. Hou, P. et al. PTBP3-mediated regulation of ZEB1 mRNA stability promotes epithelial-mesenchymal transition in breast cancer. Cancer Res. 78, 387-398 (2018)

26. Dong, R. et al. Stabilization of Snail by HuR in the process of hydrogen peroxide induced cell migration. Biochem. Biophys. Res. Commun. 356, 318-321 (2007).

27. Yoon, N. A. et al. Tristetraprolin suppresses the EMT through the downregulation of Twist1 and Snail1 in cancer cells. Oncotarget 7, 8931-8943 (2016).

28. Hamilton, K. E. et al. IMP1 promotes tumor growth, dissemination and a tumor-initiating cell phenotype in colorectal cancer cell xenografts. Carcinogenesis 34, 2647-2654 (2013).

29. Dasari, S. \& Tchounwou, P. B. Cisplatin in cancer therapy: molecular mechanisms of action. Eur. J. Pharm. 740, 364-378 (2014).

30. Lytle, N. K., Barber, A. G. \& Reya, T. Stem cell fate in cancer growth, progression and therapy resistance. Nat. Rev. Cancer 18, 669-680 (2018).

31. Wang, L. et al. Cisplatin-enriching cancer stem cells confer multidrug resistance in non-small cell lung cancer via enhancing TRIB1/HDAC activity. Cell Death Dis. 8, e2746 (2017)

32. Zhuo, W. et al. Knockdown of Snail, a novel zinc finger transcription factor, via RNA interference increases A549 cell sensitivity to cisplatin via JNK/mitochondrial pathway. Lung Cancer 62, 8-14 (2008).

33. Al-Mohanna, M. A., Al-Khalaf, H. H., Al-Yousef, N. \& Aboussekhra, A. The p16INK4a tumor suppressor controls p21WAF1 induction in response to ultraviolet light. Nucleic Acids Res. 35, 223-233 (2007).

34. Tominaga, $\mathrm{K}$. et al. Competitive regulation of nucleolin expression by HuR and miR-494. Mol. Cell Biol. 31, 4219-4231 (2011). 
35. Knopfova, L. et al. c-Myb regulates matrix metalloproteinases $1 / 9$, and cathepsin D: implications for matrix-dependent breast cancer cell invasion and metastasis. Mol. Cancer 11, 15 (2012).

36. Jurmeister, S. et al. MicroRNA-200c represses migration and invasion of breast cancer cells by targeting actin-regulatory proteins FHOD1 and PPM1F. Mol. Cell Biol. 32, 633-651 (2012).
37. Adams, C. J. et al. The p53 cofactor Strap exhibits an unexpected TPR motif and oligonucleotide-binding (OB)-fold structure. Proc. Natl Acad. Sci. USA 109, 3778-3783 (2012).

38. Ghebeh, $\mathrm{H}$. et al. Profiling of normal and malignant breast tissue show CD44high/CD24low phenotype as a predominant stem/progenitor marker when used in combination with Ep-CAM/CD49f markers. BMC Cancer 13, 289 (2013). 